\title{
Utilização de modelos de estimativa da radiação de onda longa atmosférica para Cerrado Campo Sujo
}

A radiação de onda longa atmosférica (ROL) é a variável chave para o cálculo do balanço de radiação da superfície terrestre, sendo a componente mais difícil de ser medida. Neste estudo, foi avaliado o desempenho de seis modelos que estimam ROL em dias de céu claro, parcialmente nublado e nublado, para dados obtidos na região do Cerrado Campo Sujo, na Baixada Cuiabana, em dois períodos distintos: seco (maio a agosto) e úmido (setembro a dezembro) de 2009. Dois modelos levam em consideração apenas a temperatura do ar em suas estimativas, Swinbank (1963) e de Idso et al. (1969), e os modelos de Brunt (1932), Brutsaert (1975), Idso (1981) e Bignami (1995) levam em consideração a temperatura do ar e a pressão de vapor d'água. Os seis modelos foram avaliados estatisticamente pelo coeficiente de determinação (R2) da regressão robusta e o p-valor (M-Estimador) do teste de viés dado pela regressão. Os modelos de Swinbank (1963) e de Idso et al. (1969) foram os que melhores estimaram ROL e o modelo de Idso (1981) o pior modelo estimador para o local de estudo. Sendo as equações que levam em consideração apenas a temperatura do ar em suas estimativas obtiveram melhor desempenho do que as que utilizam apenas a temperatura do ar e a pressão de vapor d'água para os dois períodos estudados, visto os valores de R2 obtidos através da regressão robusta.

\section{Use of atmospheric longwave radiation estimation models for Cerrado Campo Sujo}

\begin{abstract}
Atmospheric longwave radiation (NRW) is the key variable for calculating the earth surface radiation balance and is the most difficult component to measure. In this study, we evaluated the performance of six models that estimate OLR on clear, partly cloudy and cloudy days, for data obtained in the Cerrado Campo Sujo region, in Baixada Cuiabana, in two distinct periods: dry (May to August) and (September to December) 2009. Two models take into account only air temperature in their estimates, Swinbank (1963) and Idso et al. (1969), and the models of Brunt (1932), Brutsaert (1975), Idso (1981) and Bignami (1995) take into account air temperature and water vapor pressure. The six models were statistically evaluated by the robust regression determination coefficient (R2) and the $p$-value ( $M$ Estimator) of the regression bias test. The Swinbank (1963) and Idso et al. (1969) were the best estimators of NOR and Idso's model (1981) the worst estimator model for the study site. Since the equations that take into account only air temperature in their estimates obtained better performance than those that use only air temperature and water vapor pressure for the two periods studied, considering the R2 values obtained through the robust regression.
\end{abstract}

Keywords: Empirical models; Robust regression; Temperature.

Topic: Meteorologia, Climatologia e Mudanças Climáticas

Reviewed anonymously in the process of blind peer
Received: 02/10/2018

Approved: 03/11/2018
Jonh Billy Silva (iD)

Universidade Federal de Mato Grosso, Brasil http://lattes.cnpq.br/7620365079545430 http://orcid.org/0000-0002-5048-2795 jonhbilly9@gmail.com

Leone Francisco Amorim Curado

Universidade Federal de Mato Grosso, Brasil

http://lattes.cnpq.br/4050028967752766

leonecurado@gmail.com

Carlo Ralph Musis (iD)

Universidade de Cuiabá, Brasil

http://lattes.cnpq.br/2229878954372934

http://orcid.org/0000-0001-6047-1304

carlo.demusis@gmail.com

\author{
José de Souza Nogueira (iD) \\ Universidade Federal de Mato Grosso, Brasil \\ http://lattes.cnpq.br/5893185001802390 \\ http://orcid.org/0000-0003-2504-7924 \\ parananogueira@gmail.com \\ Thiago Rangel Rodrigues (io \\ Universidade Federal do Mato Grosso do Sul, Brasil \\ http://lattes.cnpq.br/7044858666359931 \\ http://orcid.org/0000-0003-0902-6824 \\ thiago.r.rodrigues@ufms.br
}

Referencing this:

SILVA, J. B.; CURADO, L. F. A.; MUSIS, C. R.; NOGUEIRA, J. S.; RODRIGUES, T. R.. Utilização de modelos de estimativa da radiação de onda longa atmosférica para Cerrado Campo Sujo. Revista IberoAmericana de Ciências Ambientais, v.9, n.8, p.46-56, 2018. DOI: http://doi.org/10.6008/CBPC2179-6858.2018.008.0005

DOI: 10.6008/CBPC2179-6858.2018.008.0005 


\section{INTRODUÇÃO}

A Radiação de Onda Longa Atmosférica (ROL) é oriunda da absorção e emissão da radiação de ondas curtas na atmosfera, bem como da onda longa superficial, pelos gases que compõem a atmosfera terrestre, tendo como principal absorvedor o vapor d'água (IDSO et al., 1969; DAl et al., 2014). Essa radiação está compreendida entre 4 e $100 \mu \mathrm{m}$, e embora haja instrumentos para sua medida, esses emitem radiação em comprimentos de onda e intensidade comparáveis aos da suposta medida (RANDOW et al., 2006), necessitando assim, de correções a partir da temperatura desses instrumentos, tornando-a a componente do balanço de radiação mais difícil de ser medida (CURADO et al., 2011). O instrumento que mede diretamente ROL é o pirgeômetro, que é caro e sensível (DUARTE et al., 2006) e dessa foram, se faz necessário avaliar diferentes modelos para sua estimativa.

No Brasil, a medida da radiação solar global é comum. Porém, dados disponíveis de ondas longas são raros (BLONQUIST et al., 2009). Na região do Cerrado Mato-grossense, existem poucos trabalhos e medidas regulares de ROL, mesmo sendo essa uma variável importante no cálculo do balanço de radiação à superfície, pois representa a contribuição da atmosfera e engloba informações de nebulosidade e concentração de vapor d'água (GALVÃO et al., 2000; AGUIAR, et al., 2011; CARRILHO et al., 2013). A maioria dos resultados publicados do balanço de radiação de ondas longas é estimada ou obtida como resíduo a partir da equação do balanço de radiação, tais como o estudo realizado por Carrilho et al. (2013), que avaliou e parametrizou 107 modelos para a estimativa da ROL. Outros trabalhos que podem ser citados sãos os estudos feitos por Manzi et al. (1986), Randow et al. (2006), Sicart (2010), Vilani (2010), Aguiar et al. (2011) e Guo et al. (2018).

Uma inconveniência na estimativa dessa componente é que a maioria dessas equações apenas estimam ROL para dias de céu limpo, onde essa radiação é bem simulada em função da temperatura e umidade do ar, mas as mudanças nos perfis atmosféricos de umidade do dia para o período noturno envolvem diferentes coeficientes otimizados para dados horários e diários, sendo que a emissão dessa radiação pelas nuvens pode aumentar a emissividade de céu limpo em até $55 \%$, com uma média de $20 \%$ (MOELG et al., 2009; SICART, 2010). Além disso, estimativas horárias, frequentemente exigidas em modelos de balanço de energia à superfície, são mais sujeitas a erros, apresentando melhor desempenho quando se consideram bases diárias ou médias de longo prazo (RANDOW et al., 2006; AGUIAR et al., 2011).

Logo, o objetivo deste trabalho foi estimar a radiação de onda longa proveniente da atmosfera para os períodos seco (maio a agosto) e úmido (setembro a dezembro) de 2009 na região do cerrado campo sujo e avaliar cinco modelos propostos na literatura, comparando as estimativas resultantes com valores calculados pela equação do balanço de radiação.

\section{METODOLOGIA}

\section{Área de estudo}

A região de estudo está localizada na Fazenda Miranda, nas coordenadas $15^{\circ} 43^{\prime} 53,65^{\prime \prime}$ Sul e $56^{\circ} 4^{\prime}$ 18,88" Oeste, no município de Santo Antônio de Leverger, situado na Baixada Cuiabana (MT), distante, 
aproximadamente, $15 \mathrm{~km}$ da cidade de Cuiabá, e com altitude média de $157 \mathrm{~m}$, conforme se pode visualizar na figura 1.

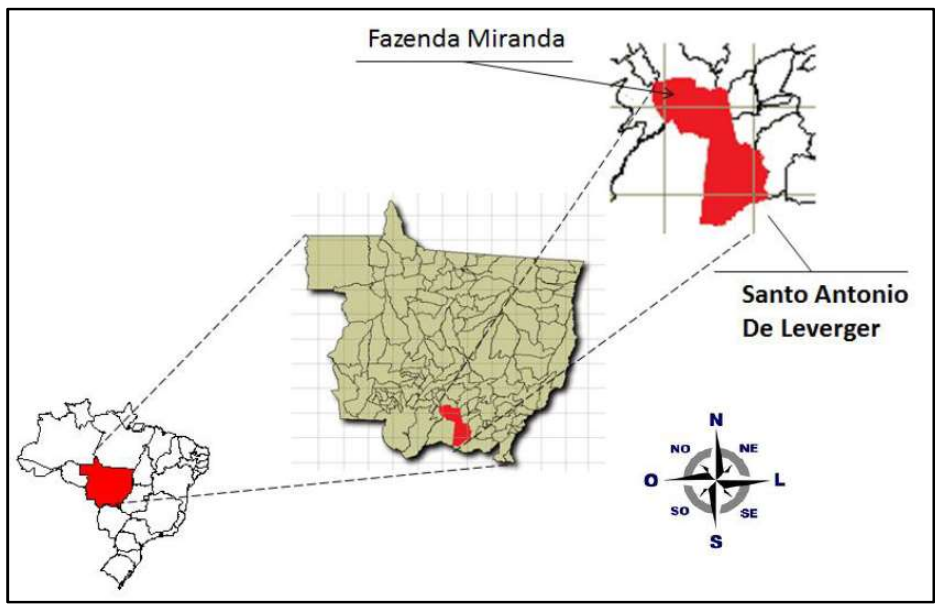

Figura 1: Mapa do Brasil; Mato Grosso, com a localização da área de estudo. Fonte: Carrilho (2011).

Segundo a classificação de Köppen, o clima de Santo Antônio do Leverger é do tipo AW, também denominado Tropical semiúmido, possui quatro a cinco meses secos e duas estações bem definidas, uma seca (outono-inverno) e uma chuvosa (primavera-verão). O local do estudo era uma pastagem florestal mista (conhecida localmente como campo sujo) que foi parcialmente desmatada há aproximadamente 35 anos (RODRIGUES et al., 2014).

A precipitação média anual e a temperatura são de $1420 \mathrm{~mm}$ e $26,5^{\circ} \mathrm{C}$, respectivamente, e a precipitação é sazonal com uma estação seca que se estende de maio a setembro (VOURLITIS et al., 2011). O intervalo de temperatura mensal média do ar é amplo em relação às florestas tropicais e subtropicais húmida, com temperatura mínima de $23,5^{\circ} \mathrm{C}$ em junho e máxima de $28,6^{\circ} \mathrm{C}$ em setembro (VOURLITIS et al., 2011). A área de pesquisa tem terreno plano a uma altitude de $157 \mathrm{~m}$ do nível do mar. O tipo de solo regional é latossolo vermelho-amarelo distrófico e rochoso, conhecido localmente como solo concrecionário distrófico (RADAMBRASIL, 1982).

\section{Instrumentação}

$\mathrm{Na}$ área de estudo, numa torre em estrutura metálica de $19 \mathrm{~m}$ de altura, com equipamentos de medições micrometeorológicas, foram coletados dados dos meses de maio a julho (período seco) e outubro a dezembro (período úmido) de 2009. As medidas de saldo de radiação (Rn) sobre a vegetação foram obtidas por meio de um saldo radiômetro NR LITE (Kipp e Zonen Delft, Inc., The Netherlands) instalado a $4 \mathrm{~m}$ de altura. A Radiação Global Incidente (RGI) e a radiação global refletida (RGR) foram medidas por meio de piranômetros LI-200X-L (Campbell Scientific, Inc., USA) instalados a 4m de altura, um com a face voltada para cima (RGI) e o outro para baixo (RGR). A Temperatura do $\operatorname{Ar}(\mathrm{TAr})$ e a Umidade Relativa do ar (UR) foram medidas por três termohigrômetros modelo HMP45AC (Vaisala, Inc., Woburn, MA, USA) instalados em três alturas, a $5 \mathrm{~m}$, a $10 \mathrm{~m}$ e a $18 \mathrm{~m}$ em relação ao nível do terreno.

Também foram utilizadas duas placas de fluxo de calor no solo modelo HFP01-L20 (Hukseflux Thermal Sensors B.V., Delft, The Netherlands) instaladas a $1 \mathrm{~cm}$ de profundidade, uma delas colocada no solo 
tipo Arenoso, e a outra colocada no solo tipo Laterita, e posteriormente calculada uma média dos fluxos. Uma placa de fluxo de calor HFP01 (Rukseflux, Inc., The Netherlands), um pluviômetro de báscula modelo TR-525M (Texas Eletronics, Inc., Dallas, TX, USA) instalado a 5m de altura do solo e um anemômetro de conchas 03101-L Wind Sentry Anemometer (RM Young, Inc., USA). Os dados micrometeorológicos foram coletados por meio de sensores de aquisição de dados, ligados a um datalogger CR 1000 (Campbell Scientific, Inc., USA), com intervalo de leitura de 30 segundos e registro das médias a cada 30 minutos

\section{Índice de claridade (Kg)}

Como todos os modelos testados foram desenvolvidos para situação de céu sem nebulosidade, para se tentar atingir uma melhor performance, buscou-se separar os dados em dias de céu limpo, mas devido à baixa ocorrência de dias nesta situação, juntou-se a essa classificação, os dias parcialmente nublados e nublado. A cobertura do céu foi determinada pelo índice de claridade (Kt), conforme se tem na equação proposta, onde entre o intervalo de $\mathrm{Kt} \leq 0,3$ é considerado céu nublado, entre 0,3 $<\mathrm{Kt}<0,65$ céu parcialmente nublado e entre 0,65 < Kt < 1,0 céu limpo (RENSHENG, 2004; VILANI, 2010; IQBAL, 2012). Tem-se $K_{t}=\frac{R g}{R_{0}}$, em que Rg é a radiação solar global ( $\left.\mathrm{MJ} \mathrm{m}^{-2} \mathrm{dia}^{-1}\right)$; e Ro é radiação solar que atinge o topo da atmosfera em MJ $\mathrm{m}^{-2} \mathrm{dia}^{-1}$.

Tudo isso sendo estimada pela equação seguinte, que depende da latitude $(\varphi)$, da correção da excentricidade da órbita terrestre ( $d r)$ da equação posterior, da declinação solar $(\delta)$, sendo essa prevista na quarta equação 4, e do ângulo horário $(\mathrm{H})$, proposto na quinta equação. Tem-se as seguintes equações: $R_{o}=$

$37,6 \cdot d r\left(\frac{\pi}{180} \cdot H \cdot \operatorname{sen} \phi \cdot \operatorname{sen} \delta+\cos \phi \cdot \cos \delta \cdot \operatorname{sen} H\right) ; \quad d r=1+0,033 \cdot \cos \left(\frac{360 \cdot D J}{365}\right) ; \quad \delta=$ $23,45 \cdot \operatorname{sen}\left[\frac{360}{365}(D J+284)\right] ; H=\cos ^{-1}(-\tan \varphi \cdot \tan \delta)$.

\section{Estimativa da radiação de ondas longas atmosféricas (ROL)}

A equação geral para a estimativa da ROL é a de Stefan-Boltzmann, que considera a emissividade $(\varepsilon)$ do $\operatorname{ar}$ (KRUK, 2010; HERRERO, 2012; GUO et al., 2018). Tem-se $R O L=\sigma . \varepsilon . T_{a r}{ }^{4}$, em que $\sigma=5,67051 \cdot 10^{-8} \mathrm{~W}$ $\mathrm{m}^{-2} \mathrm{~K}^{-4}$ é a constante de Stefan-Boltzmann e Tar $(\mathrm{K})$ é a temperatura do ar. Neste trabalho foram testados seis modelos, que se aplicam na faixa de temperatura dos locais estudados (tabela 1). Os dois primeiros modelos utilizados para estimativa da ROL, foram os propostos por Swinbank (1963) e Idso et al. (1969), que foram escolhidos por levar em consideração em suas estimativas apenas Tar dentro de uma faixa de 2 a $29^{\circ} \mathrm{Ce}-29$ a $37^{\circ} \mathrm{C}$, respectivamente.

Ainda na tabela 1, têm-se os modelos propostos por Brutsaert (1975), Brunt (1932), Bignami (1995) e Idso (1981) escolhidos por atuarem na faixa de temperatura entre -40 e $45^{\circ} \mathrm{C}$ e levarem em consideração a pressão de vapor d'água (ea) em hPa, proposto na segunda equação (IDSO, 1981; PRATA, 1996; DUARTE et al., 2006).

Como a atmosfera é um sistema complexo, o vapor d'água que é considerado um dos principais gases absorvedores e emissores de ROL, torna-se peça fundamental no balanço radiativo da Terra, devendo, assim, 
ser levado em consideração na estimativa da ROL (RÄDEL et al., 2015), onde se tem $e_{a}=\frac{e_{s} \text {. UR }}{100}$, em que ea é a pressão de saturação do vapor d'água atmosférico determinada pela Equação de Tetens (TETENS, 1930), associando-se à equação $e_{S}=6,1078 \cdot 10^{\frac{7,5 \cdot T_{a r}}{273,3+T_{a r}}}$. A diferença entre a pressão exercida pela quantidade de vapor d'água existente no ar (es) e a pressão máxima que pode ser alcançada (ea), é denominada de déficit de pressão de saturação de vapor d'água no ar (DVP).

Tabela 1: Equações utilizadas para estimar a radiação de onda longa atmosférica, em que $\sigma$ é a constante de StefanBoltzmann $\left(5,6697.10^{-8} \mathrm{~W} \mathrm{~m}^{-2} \mathrm{~K}^{-4}\right)$, ea a pressão de vapor d'água (hPa), Tar a temperatura do ar (K) próximo a superfície.

\begin{tabular}{|l|l|}
\hline \multicolumn{1}{|c|}{ Modelo } & \multicolumn{1}{c|}{ Equação } \\
\hline Brunt (1932) & $\mathrm{ROL}=\left(0,52+0,065 \sqrt{e_{a}}\right) \sigma T_{a r}{ }^{4}$ \\
\hline Swinbank (1963) & $\mathrm{ROL}=\left(9,2.10^{-6}\right) \sigma T_{a r}{ }^{6}$ \\
\hline Idso \& Jackson (1969) & $\mathrm{ROL}=\left\{1-0,26 \exp \left[-7,77.10^{-4}\left(273-T_{a r}\right)\right]\right\} \sigma T_{a r}{ }^{4}$ \\
\hline Brutsaert (1975) & $\mathrm{ROL}=\left[1,24\left(e_{a} / T_{a r}\right)^{1 / 7}\right] \sigma T_{a r}{ }^{4}$ \\
\hline Idso (1981) & $\mathrm{ROL}=\left[0,7+5,95.10^{-5} e_{a} \exp \left(1500 / T_{a r}\right)\right] \sigma T_{a r}{ }^{4}$ \\
\hline Bignami et al. (1995) & $\mathrm{ROL}=\left(0,684+0,0056 e_{a}\right) \sigma T_{a r}{ }^{4}$ \\
\hline
\end{tabular}

\section{Análise estatística}

O desempenho das equações utilizadas para estimar a emissividade atmosférica foi avaliado estatisticamente através do Desvio Médio Absoluto (DMA), proposto na próxima primeira equação que segue, Desvio Médio Quadrático (DMQ), que se pode visualizar na equação seguinte, coeficiente de determinação $\left(R^{2}\right)$ da regressão robusta e o $p$-valor (M-Estimador) do teste de viés dado pela regressão.

Tem-se $D M A=\frac{1}{N} \sum_{i=1}^{N}\left|y_{i}-x_{i}\right|$ e $D M Q=\left[\frac{1}{N} \sum_{i=1}^{N}\left(y_{i}-x_{i}\right)^{2}\right]^{1 / 2}$, em que $\mathrm{N}$ é o número de dados da amostra; y refere-se aos valores estimados; e $\mathrm{x}$ aos valores calculados e a barra acima da variável corresponde ao valor médio da variável. Optou-se pelo uso da regressão robusta por ser esta considerada uma técnica adequada não somente com respeito presença de outliers, mas também com relação a heterocedásticidade (CUNHA et al., 2002). Utilizou-se das bibliotecas mass e robust disponíveis no repositório padrão do $\mathrm{R}$.

\section{RESULTADOS}

\section{Análise das variações sazonais da temperatura do ar, umidade relativa do ar e do déficit da pressão de} vapor d'água

A temperatura média mensal do ar para o período seco estudado variou entre $11,22^{\circ} \mathrm{C}$ e $31,95^{\circ} \mathrm{C}$, com média de $26,03^{\circ} \mathrm{C}$. Já no período úmido esses valores oscilaram entre $17,58^{\circ} \mathrm{C}$ e $34,22^{\circ} \mathrm{C}$ com média de $28,33^{\circ} \mathrm{C}$ (figura 2 ). A umidade relativa oscilou entre $31,56 \%$ e $94,31 \%$ no período seco e entre $35,21 \%$ e 94,75\% para período úmido com médias de 60,59\% e 64,99\% respectivamente, não apresentando um padrão definido durante o ano, sendo que, na estação seca, seu valor decai em comparação com a estação úmida. Isso é devido à regularidade de chuvas dessa época durante os meses de setembro a março e ausência de chuvas nos meses de maio a agosto. Assim, como no período úmido, houve valores maiores da umidade 
relativa e de temperatura, se comparados com o período seco, espera-se que a emissividade atmosférica seja maior no período úmido do que no seco.

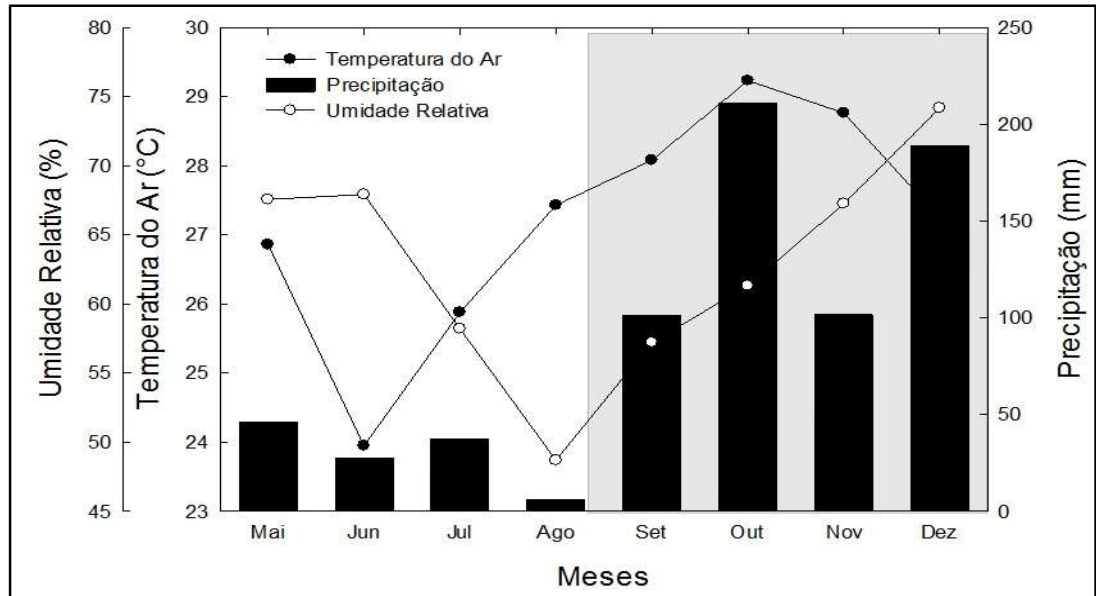

Figura 2: Valores médios mensais da temperatura do ar, umidade relativa do ar e precipitação média acumulada em 2009. A área sombreada no gráfico representa o período úmido, ou os meses consecutivos, quando a precipitação é > $100 \mathrm{~mm}$.

A precipitação obteve valores médios de $29,38 \mathrm{~mm}$ de valor acumulado para o período seco e 149,08mm de valor acumulado para o período úmido. O mês de agosto teve o menor valor médio de precipitação, apenas $6,20 \mathrm{~mm}$ de chuva acumulada, e o mês de outubro foi o de maior valor de chuva acumulada, $211 \mathrm{~mm}$. Esses valores são bem próximos ao encontrado por Carrilho Sobrinho et al. (2013) para a mesma área em estudo considerando apenas os meses de junho, julho, outubro e novembro. 0 maior déficit de pressão de vapor d'água (DPV) ocorreu no mês de outubro (16,66 hPa), início do período úmido. Já o menor valor para o DPV foi encontrado no mês de dezembro $(9,65 \mathrm{hPa})$ (figura 3).

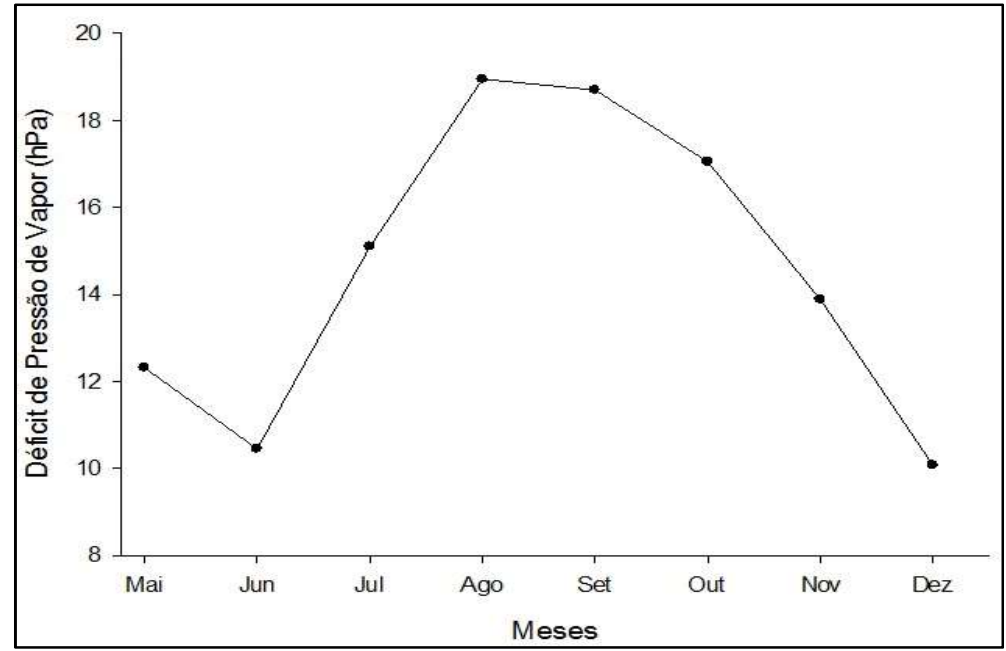

Figura 3: Valores médios mensais do déficit de pressão de vapor d'água (DPV) para Fazenda Miranda nos meses de maio a julho e outubro a dezembro.

Esses valores estão bem próximos aos valores encontrado por Aguiar et al. (2011), 16,66hPa para pastagem e 15,31hPa para floresta amazônica no período entre junho a agosto. Randow et al. (2006) relata que mesmo após um longo período de seca, a floresta pode manter uma grande retirada de água do solo. 
Por outro lado, as gramíneas sofrem mais com grandes períodos sem precipitação, uma vez que seu sistema radicular é mais curto (AGUIAR et al., 2011), similar a vegetação do cerrado estudado.

\section{Estimativa da radiação de onda longa atmosférica}

Durante o período estudado (235 dias), houve maior percentual para os dias de céu limpo (45,10\%), seguido por dias de céu parcialmente nublado $(41,28 \%)$ e dias de céu nublados $(13,62 \%)$. Os dias de céu limpo se concentraram no período seco. Já no período úmido a maioria dos dias foram parcialmente nublado. A tabela 3 apresenta as estatísticas correspondentes, mostrando que os valores (negativos) do desvio médio absoluto (DMA) variaram de $-30,01 \mathrm{Wm}^{-2}$ a $-125,44 \mathrm{Wm}^{-2}$.

Isso indica que para a maioria das equações propostas na literatura, os resultados estimados foram inferiores aos medidos, independentemente de cada equação levar em consideração a pressão de vapor e a temperatura do abrigo, ou somente a temperatura do abrigo. As figuras 4 e 5 apresentam os valores de ROL estimado com cada modelo, a partir das equações originais mostradas na tabela 1. Observa-se que os pontos de cada gráfico contido nestas figuras se ajustam a uma reta para os dois períodos.

Tabela 2: Contagem de dias de céu nublado, parcialmente nublados e céu limpo e média de radiação de ondas longa atmosférica.

\begin{tabular}{|c|c|c|c|c|c|c|c|c|c|}
\hline \multicolumn{10}{|c|}{ Radiação de Onda Longa Atmosférica $\left(\mathrm{Wm}^{-2}\right)$} \\
\hline Mês & $\begin{array}{l}\text { Cobertura do } \\
\text { Céu }\end{array}$ & Dias & $\begin{array}{c}\text { Balanço de } \\
\text { Radiação }\end{array}$ & $\begin{array}{c}\text { Idso \& } \\
\text { Jackson } \\
\text { (1969) }\end{array}$ & $\begin{array}{l}\text { Swinbank } \\
\text { (1963) }\end{array}$ & $\begin{array}{c}\text { Brutsaert } \\
\text { (1975) }\end{array}$ & $\begin{array}{l}\text { Brunt } \\
\text { (1932) }\end{array}$ & $\begin{array}{c}\text { Bignami } \\
\text { (1995) }\end{array}$ & $\begin{array}{l}\text { Idso } \\
\text { (1981) }\end{array}$ \\
\hline \multirow{3}{*}{ Maio } & Céu Limpo & 23 & 452,46 & 346,52 & 396,30 & 409,95 & 398,82 & 388,98 & 431,15 \\
\hline & Par. Nublado & 6 & 426,96 & 327,39 & 363,45 & 388,90 & 378,07 & 368,63 & 416,22 \\
\hline & Nublado & 2 & 405,95 & 311,61 & 336,78 & 363,08 & 349,92 & 342,33 & 388,42 \\
\hline \multirow{3}{*}{ Junho } & Céu Limpo & 21 & 437,11 & 335,01 & 376,44 & 386,69 & 373,07 & 365,91 & 404,75 \\
\hline & Par. Nublado & 5 & 404,67 & 310,64 & 335,27 & 357,70 & 343,71 & 337,35 & 380,39 \\
\hline & Nublado & 4 & 404,06 & 310,19 & 334,48 & 359,27 & 345,80 & 338,97 & 383,71 \\
\hline \multirow{3}{*}{ Julho } & Céu Limpo & 26 & 444,74 & 340,73 & 386,30 & 392,16 & 378,16 & 371,05 & 408,28 \\
\hline & Par. Nublado & 3 & 416,72 & 319,67 & 351,40 & 363,68 & 349,51 & 344,59 & 382,25 \\
\hline & Nublado & 2 & 385,83 & 296,45 & 312,63 & 332,44 & 317,42 & 314,36 & 353,10 \\
\hline \multirow{3}{*}{ Agosto } & Céu Limpo & 27 & 425,97 & 326,56 & 364,71 & 368,95 & 353,78 & 349,06 & 386,10 \\
\hline & Par. Nublado & 2 & 351,38 & 270,43 & 273,15 & 308,17 & 293,71 & 289,21 & 339,21 \\
\hline & Nublado & 2 & 366,19 & 281,56 & 291,79 & 330,70 & 318,55 & 311,36 & 365,66 \\
\hline \multirow{3}{*}{ Setembro } & Céu Limpo & 9 & 489,14 & 359,38 & 419,28 & 416,12 & 402,70 & 394,38 & 429,12 \\
\hline & Par. Nublado & 17 & 472,56 & 347,45 & 398,30 & 406,26 & 394,04 & 385,35 & 424,44 \\
\hline & Nublado & 4 & 429,55 & 316,46 & 344,98 & 363,47 & 349,77 & 343,91 & 385,30 \\
\hline \multirow{3}{*}{ Outubro } & Céu Limpo & 0 & & & & & & & \\
\hline & Par. Nublado & 28 & 480,40 & 353,09 & 407,94 & 419,22 & 408,75 & 398,50 & 440,14 \\
\hline & Nublado & 3 & 463,45 & 340,90 & 386,46 & 407,04 & 397,00 & 386,68 & 432,58 \\
\hline \multirow{3}{*}{ Novembro } & Céu Limpo & 0 & 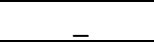 & & & & $=$ & & \\
\hline & Par. Nublado & 24 & 480,14 & 352,91 & 407,56 & 423,98 & 415,10 & 404,03 & 448,46 \\
\hline & Nublado & 6 & 458,35 & 337,23 & 380,17 & 405,16 & 395,94 & 385,41 & 433,94 \\
\hline \multirow{3}{*}{ Dezembro } & Céu Limpo & 0 & & & & & & & \\
\hline & Par. Nublado & 12 & 473,24 & 347,95 & 398,79 & 418,01 & 409,00 & 398,09 & 443,81 \\
\hline & Nublado & 9 & 456,82 & 336,12 & 378,23 & 405,20 & 396,44 & 385,76 & 435,59 \\
\hline
\end{tabular}

Legenda: (-) significa que não houve estimativa de radiação de ondas longa atmosféricas. Fonte: Brunt (1932);

Swinbank (1963); Idso et al. (1969); Brutsaert (1975); Idso (1981); Bignami (1995).

Tabela 3: Estatísticas relacionando os valores estimados da radiação de ondas longa atmosférica (ROL) nos períodos seco e úmido.

\begin{tabular}{|c|c|c|c|c|}
\hline \multicolumn{5}{|c|}{ Período Seco } \\
\hline Equação & DMA (Wm $\left.\mathbf{~}^{-2}\right)$ & DMQ $\left(\mathbf{W m}^{-2}\right)$ & $\mathbf{R}^{\mathbf{2}}$ & M-Estimador \\
\hline Idso e Jackson & $-61,23$ & 61,83 & $0,62^{*}$ & $15,57^{*}$ \\
\hline
\end{tabular}




\begin{tabular}{|c|c|c|c|c|}
\hline Swinbank & $-49,55$ & 50,47 & $0,61^{*}$ & $11,18 * *$ \\
\hline Brutsaert & $-63,02$ & 64,02 & $0,57 *$ & $6,11 * * *$ \\
\hline Brunt & $-70,05$ & 70,68 & $0,56^{*}$ & 4,34 \\
\hline Bignami & $-30,01$ & 34,17 & $0,62 *$ & 2,58 \\
\hline Idso & $-61,23$ & 61,83 & $0,50 *$ & 3,77 \\
\hline \multicolumn{5}{|c|}{ Período Úmido } \\
\hline Equação & $\mathrm{DMA}\left(\mathrm{Wm}^{-2}\right)$ & $\mathrm{DMQ}\left(\mathrm{Wm}^{-2}\right)$ & $\mathbf{R}^{2}$ & M-Estimador \\
\hline Idso e Jackson & $-125,44$ & 125,55 & 0,73 & $12,34 * *$ \\
\hline Swinbank & $-74,12$ & 74,27 & 0,73 & $11,01 * *$ \\
\hline Brutsaert & $-60,04$ & 60,54 & 0,57 & $8,43 * * *$ \\
\hline Brunt & $-70,42$ & 71,08 & 0,44 & $6,03 * * *$ \\
\hline Bignami & $-80,42$ & 80,85 & 0,51 & $6,17^{* * *}$ \\
\hline Idso & $-37,55$ & 39,85 & 0,16 & $9,98 * *$ \\
\hline
\end{tabular}

Legenda: $*, * * * * *$ Significativo a 0,001, 0,01 e 0,05 de probabilidade, respectivamente.

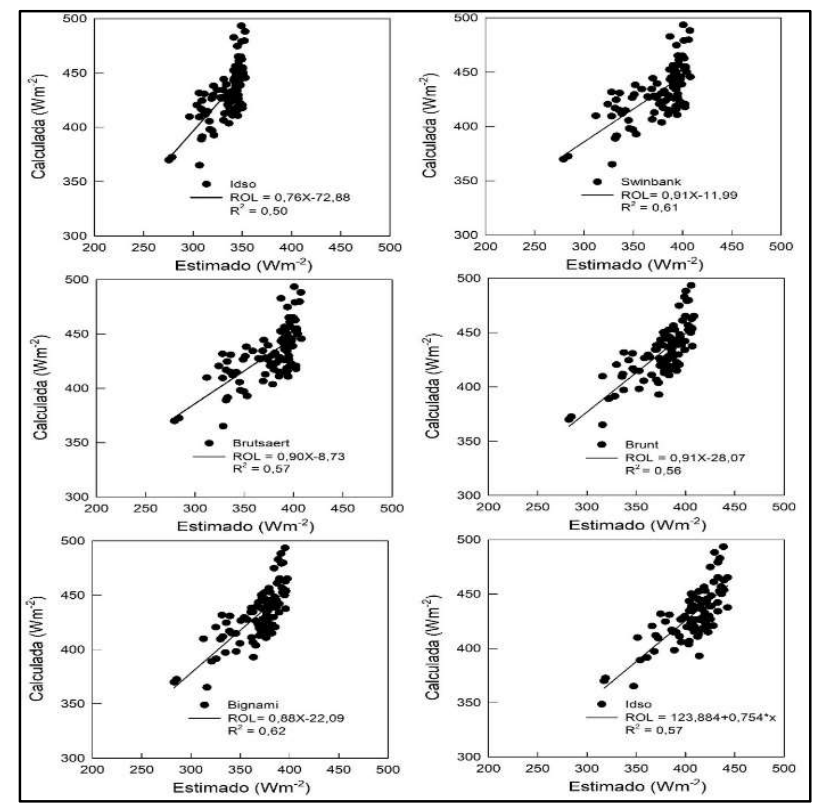

Figura 4: Comparação entre os valores calculadas de ROL e os valores estimados para os meses de maio a agosto para Fazenda Miranda. Fonte: Brunt (1932); Swinbank (1963), Idso et al. (1969); Brutsaert (1975); Idso (1981); Bignami (1995).

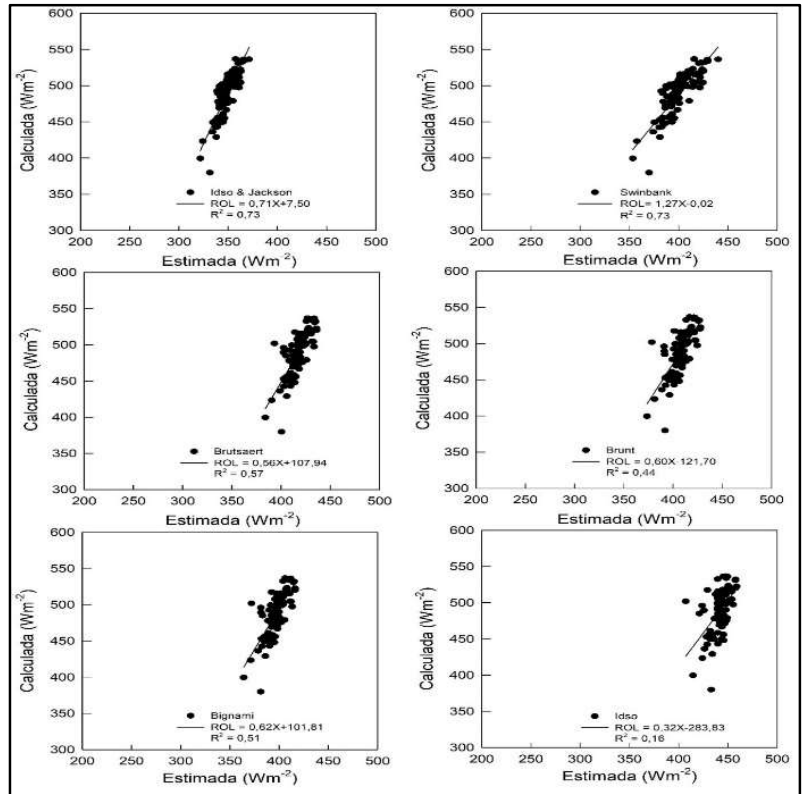

Figura 5: Comparação entre os valores medidos de ROL e os valores estimados para os meses de setembro a dezembro para Fazenda Miranda. Fonte: Brunt (1932); Swinbank (1963); Idso et al. (1969); Brutsaert (1975); Idso (1981); Bignami (1995).

Ao analisar os ciclos médios diários da radiação de onda longa atmosférica calculada e estimada pelos modelos, nota-se que as equações subestimam a radiação de onda longa atmosférica ao longo do dia no período seco, conforme se pode visualizar na figura 6, denominada 'Ciclo médio diário da radiação de onda longa atmosférica observada e estimada para os meses de maio a agosto, B: para os meses de setembro a dezembro'.

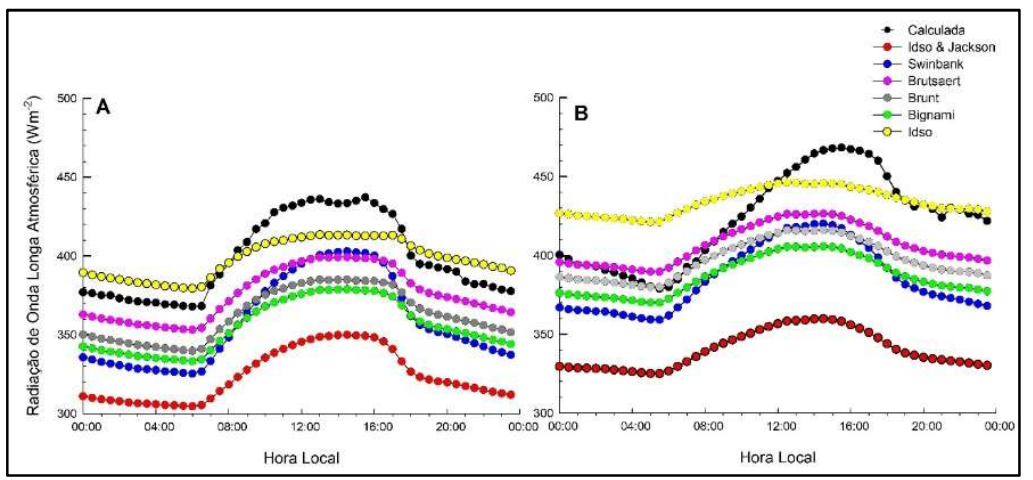

Figura 6: Ciclo médio diário da radiação de onda longa atmosférica observada e estimada. A: para os meses de maio a agosto, B: para os meses de setembro a dezembro. Fonte: Brunt (1932); Swinbank (1963); Idso et al. (1969); Brutsaert (1975); Idso (1981); Bignami (1995). 


\section{DISCUSSÃO}

Os valores das médias mensais de ROL estimados variaram desde $270,43 \mathrm{~W} \mathrm{~m} \mathrm{~m}^{-2}$ até $489,14 \mathrm{~W} \mathrm{~m} \mathrm{~m}^{-2}$, onde entre os métodos o de Idso (1981) obteve o maior valor, e do método de Idso et al. (1969) o de menor valor. A maior média mensal ROL foi estimada pelo método de Idso $\left(448,46 \mathrm{~W} \mathrm{~m}^{-2}\right)$ seguida de Brutsaert $\left(423,98 \mathrm{~W} \mathrm{~m}^{-2}\right)$, Brunt $\left(415,10 \mathrm{~W} \mathrm{~m}^{-2}\right)$, Swinbank $\left(407,94 \mathrm{~W} \mathrm{~m}^{-2}\right)$ e Idso e Jackson $\left(353,09 \mathrm{~W} \mathrm{~m}^{-2}\right)$ (Tabela 2$)$.

Espera-se obter valores mais elevados de ROL em dias nublados, já que as nuvens são as maiores contribuidoras de ROL para a superfície, pois irradiam como corpo negro $(\varepsilon \approx 1)$ a partir da base das nuvens (ARYA, 2001; VILANI et al., 2010), o que concorda com nossos dados, pois os maiores valores observados foram para o período úmido, que teve a maioria dos dias parcialmente nublados. Após as estimativas, os valores de ROL foram separados por período e calculou-se a Desvio Médio Absoluto (DMA), Desvio Médio Quadrático (DMQ), o coeficiente de determinação $\left(R^{2}\right)$ e o $M$-Estimador dado pela regressão robusta como mostrado na tabela 3.

Maiores erros nos meses referentes ao período seco demonstram que essas equações não se ajustam bem a local de estudo, em que a variação de DMA $\left(-30,01\right.$ a -63,02), DMQ $\left(34,17\right.$ a 70,68), $R^{2}(0,50$ a 0,62) foi maior do que a variação no período úmido, para DMA $(-37,55$ a -125,44), DMQ $(39,85$ a 125,55) e R² $(0,16$ a $0,73)$. Essa tendência de subestimativa pela maioria das equações também foi encontrada nos trabalhos de Randow et al. (2006) e Aguiar et al. (2011), que indicaram que o problema poderia estar relacionado aos coeficientes utilizados nas equações, os quais foram ajustados para outras regiões.

Os valores do desvio médio quadrático (DMQ) obtidos foram elevados. Esses valores de DMA e de DMQ encontrados foram maiores que aqueles encontrados por Galvão et al. (2000), para a área de pastagem, Correia (2000) em uma região de policultivo na Amazônia, Randow et al. (2006), para o Pantanal Sul Matogrossense e Aguiar et al. (2011) para floresta e pastagem no sudoeste amazônico, o que mostra que os coeficientes ajustam-se menos às condições do local de estudo, o que pode estar relacionado com o fato dos locais onde os estudos citados, estarem situado em latitude mais próxima daquelas para as quais os modelos foram originalmente desenvolvidos. A melhor adequação em função da latitude foi em função da temperatura da região e, mais fortemente, da umidade do ar, que na Amazônia é muito alta, diferentemente do cerrado campo sujo nos dois períodos.

Pode-se observar, ainda pela tabela 3, que os modelos apresentaram um melhor resultado para o período úmido, tendo em vista as condições de nebulosidade. Com 54,90\% dias de céu nublado e parcialmente nublado, é esperado o aumento de ROL, já que quanto maior for a nebulosidade o desempenho dos modelos aumenta. Os métodos de Idso e Jackson e Bignami foram as que obtiveram os maiores valores de $R^{2}(0,62)$ e o método de Swinbank obteve o valor de 0,61 para o período seco. Já para o período úmido, os métodos de Idso e Jackson e Swinbank obtiveram o valor de $R^{2}$ de 0,73 e o método de Brutsaert de 0,57.

Em relação ao ciclo médio diário, as equações subestimam $\mathrm{ROL}$ ao longo do dia no período seco (Figura 6A), concordando com Aguiar et al. (2011), onde os autores relatam que as equações que levam em 
consideração apenas a temperatura do ar, foram as que mais subestimaram a radiação de onda longa atmosférica, relacionadas com os altos valores do déficit de pressão de vapor d'água desse período.

Por sua vez, essas mesmas equações que levam em consideração apenas a temperatura do ar, se mostraram mais eficiente nos meses mais úmidos (figura 6B), onde ocorrem menores amplitudes térmicas, enquanto que nos mais secos se percebe maiores erros nas estimativas, o que demonstra a sensibilidade dessas equações à variação da temperatura do ar, uma vez que a pressão de vapor d'água praticamente não varia ao longo do dia.

Deste modo, a oscilação dos valores de ROL ao longo dos períodos justifica-se por efeitos da sazonalidade da região. Os meses considerados secos apresentam baixos índices de UR, além de pouco registro pluviométrico, com menor concentração de nuvens e, por consequência, menos emissão de onda longa atmosférica, uma vez que, o vapor d'agua é o um dos principais gases absorvedores e emissores de ROL (RÄDEL et al., 2015). Já os meses com valores altos de UR e registro pluviométrico, obtiveram maiores valores de ROL.

\section{CONCLUSÕES}

Avaliou-se o desempenho de seis modelos para estimativa de ROL na região do Cerrado Campo Sujo. O desempenho dos modelos concordou com os valores de ROL calculados pela equação do balanço de radiação, apesar de seus coeficientes serem específicos para as condições ambientais dos locais para os quais foram desenvolvidos. As equações que levam em consideração apenas a temperatura do ar em suas estimativas obtiveram melhor desempenho do que as que utilizam a temperatura do ar e a pressão de vapor d'água para os dois períodos estudados, visto os valores de $\mathrm{R}^{2}$ obtidos através da regressão robusta. Os modelos de Swinbank (1963) e de Idso et al. (1969) foram os que melhor estimaram ROL, e o modelo de Idso (1981), o pior modelo estimador para o local de estudo.

\section{REFERÊNCIAS}

AGUIAR, L. J. G.; FISCH, G. R.; FERREIRA, W. P. M.; COSTA, A. C. L. D.; COSTA, J. M. N. D.; AGUIAR, R. G.. Estimativa da radiação de onda longa atmosférica em áreas de floresta e de pastagem no sudoeste da Amazônia. Revista Brasileira de Meteorologia, São Paulo, v.26, n.2, p.215-224, 2011.

ARYA, P. S.. Introduction to micrometeorology. Amsterdam: Elsevier, 2001.

BIGNAMI, F.; MARULLO, S.; SANTOLERI, R.; SCHIANO, M. E.. Longwave radiation budget in the Mediterranean Sea. Journal of Geophysical Research, Oceans, v.100, n.2, p.2501-2514, 1995.

BLONQUIST, J. M.; TANNER, B. D.; BUGBEE, B.. Evaluation of measurement accuracy and comparison of two new and three traditional net radiometers. Agricultural and Forest Meteorology, Amsterdam, v.149, p.1709-1721, 2009.

BRUNT, D.. Notes on radiation in the atmosphere. Quarterly Journal Royal Meteorological Society, v.58, n.247, p.389418, 1932.
BRUTSAERT, W.. On a derivable formula for long-wave radiation from clear skies. Water Resources Research, Washington, v.11, n.5, p.742-744, 1975.

CARRILHO SOBRINHO, F. J.; PAULA, C. S. B.; SOUZA N. J.; BORGES, P. J. O.. Modelos para estimativa da radiação de onda longa atmosférica no cerrado Mato-

Grossense. Monografias Ambientais, v.12, n.12, p.27342748, 2013.

CORREIA, F. W. S.. Estudo do Balanço de Radiação em Área de Policultivo na Amazônia. Dissertação (Mestrado em Meteorologia) - Instituto Nacional de Pesquisas Espaciais, São José dos Campos, 2000.

CUNHA, U. S.; MACHADO, S. A.; FIGUEIREDO FILHO, A.. Uso de análise exploratória de dados e de regressão robusta na avaliação do crescimento de espécies comerciais de terra firme da Amazônia. Brasília: Embrapa, 2002. 
CURADO, L. F. A.; RODRIGUES, T. R.; BIUDES, M. S.; PAULO, S. R.; PAULO, I. J. C.; NOGUEIRA, J. S.. Estimativa sazonal da emissividade atmosférica através da Equação de Brutsaert no norte do Pantanal Mato-grossense. Ciência e Natura, v.33, n.2, p.167, 2011.

DAI, Q.; FANG, X.. A simple model to predict solar radiation under clear sky conditions. Advances in Space Research, v.53, n.8, p.1239-1245, 2014. DOI:

http://doi.org/10.1016/i.asr.2014.01.025

DUARTE, H. F.; DIAS, N. L.; MAGGIOTTO, S. R.. Assessing daytime downward longwave radiation estimates for clear and cloudy skies in southern Brazil. Meteorologia Agrícola e Florestal, v.139, p.171-181, 2006.

EMBRAPA. Empresa Brasileira de Pesquisa Agropecuária. Sistema brasileiro de classificação de solos. Brasília: Embrapa, 1999.

GALVÃO, J. A. C.; FISCH, G.. Balanço de Radiação em Áreas de pastagem na Amazônia. Revista Brasileira de Agrometeorologia, v.8, n.1, p.1-10, 2000.

GUO, Y.; CHENG, J.; LIANG, S.. Comprehensive assessment of parameterization methods for estimating clear-sky surface downward longwave radiation. Theoretical and Applied Climatology, p.1-14, 2018. DOI: http://doi.org/10.1007/s00704-018-2423-7

HERRERO, J.; POLO, M. J.. Parameterization of atmospheric longwave emissivity in a mountainous site for all sky conditions. Hydrology and Earth System Sciences, v.16, n.9, p.3139, 2012

IDSO, S. B.. A set of equations for full spectrum and 8 to 14 ìm and 10.5 to $12.5 \mu \mathrm{m}$ thermal radiation from cloudless skies. Water Resources Research, v.17, n.2, p.295-304, 1981.

IDSO, S. B.; JACKSON, R. D.. Thermal radiation from atmosphere. Journal Geophysical Research, Washington, v.74, n.23, p.5397-5403, 1969.

IQBAL, M.. An introduction to solar radiation. Amsterdam: Elsevier, 2012.

KRUK, N. S.; VENDRAME, Í. F.; ROCHA, H. R.; CHOU, S. C.; CABRAL, O.. Downward longwave radiation estimates for clear and all-sky conditions in the Sertãozinho region of São Paulo, Brazil. Theoretical and Applied Climatology, v.99, n.2, p.115-123, 2010.
MACHADO, N. G.; MEIRELLES, T.; DANELICHEN, V. H. M.; QUERINO, C. A. S.; BIUDES, M. S.. Estimation of Rainfall by neural network over a neotropical region. Revista Brasileira de Climatologia, v.17, p.44-54, 2015. DOI: http://doi.org/10.5380/abclima.v17i0.40799

MOELG, T.; CULLEN, N. J.; KASER, G.. Solar radiation, cloudiness and longwave radiation over low-latitude glaciers: implications for mass-balance modelling. Journal of Glaciology, v.55, n.190, p.292-302, 2009.

PRATA, A. J.. A new long-wave formula for estimating downward clear: sky radiation at the surface. Quartely Journal of the Royal Meteorologica Society, v.122, p.11271151,1996

RÄDEL, G.; SHINE, K. P.; PTASHNIK, I. V.. Global Radiative and Climate Effect of the Water Vapour Continuum at Visible and Near-Infrared Wavelengths. Quartely Journal of the Royal Meteorology Society, v.141, p.727-738, 2015. DOI: https://doi.org/10.1002/qj.2385

RANDOW, R. C. S.; AVALÁ, R. C. S.. Estimativa da radiação de onda longa atmosférica no pantanal sul mato-grossense durante os períodos secos de 1999 E 2000. Revista Brasileira de Meteorologia, v.21, n.3, p.398-412, 2006.

SICART, J. E.; HOCK, R.; RIBSTEIN, P.; CHAZARIN, J. P.. Sky longwave radiation on tropical Andean glaciers: parameterization and sensitivity to atmospheric variables. Journal of Glaciology, v.56, n.199, p.854-860, 2010.

SWINBANK, W. C.. Long-Wave Radiation from clear skies. Quarterly Journal of the Royal Meteorological Society, v.89, n.381, p.339-348, 1963.

TETENS, O.. Uber cinige meterorologische Begriffe. Surveys in Geophysics, n.6, p.297-309, 1930.

VILANI, M. T.; SANCHES, L.; PINHEIRO, M. R.; BORGES, P. J. O.. Estimativa da radiação de onda longa incidente em uma floresta semidecídua tropical da Bacia Amazônica. Ciência e Natura, v.32, n.1, 2010.

WILLMOTT, C. J.; ACKLESON, S. G.; DAVIS, J. J.; FEDDEMA, K. M.; KLINK, D. R.. Statistics for the evaluation and comparison of models. Journal of Geophysical Research, v.90, p.89959005, 1985

A CBPC - Companhia Brasileira de Produção Científica (CNPJ: 11.221.422/0001-03) detém os direitos materiais desta publicação. Os direitos referem-se à publicação do trabalho em qualquer parte do mundo, incluindo os direitos às renovações, expansões e disseminações da contribuição, bem como outros direitos subsidiários. Todos os trabalhos publicados eletronicamente poderão posteriormente ser publicados em coletâneas impressas sob coordenação da Sustenere Publishing, da Companhia Brasileira de Produção Científica e seus parceiros autorizados. Os (as) autores (as) preservam os direitos autorais, mas não têm permissão para a publicação da contribuição em outro meio, impresso ou digital, em português ou em tradução. 